\title{
INOVATIVIDADE, ENVOLVIMENTO, ATITUDE E EXPERIÊNCIA NA ADOÇÃO DA COMPRA ON-LINE
}

\author{
INNOVATION, INVOLVEMENT, ATTITUDE AND EXPERIENCE IN BUYING ONLINE \\ INNOVACIÓN, PARTICIPACIÓN, ACTITUD Y EXPERIENCIA EN LA ADOPCIÓN DE LA COMPRA ONLINE
}

\section{RESUMO}

Este trabalho procura analisar as inter-relações entre a inovatividade, o envolvimento, a atitude dentro do modelo Theory of Planned Behavior (TPB) decomposto desenvolvido na psicologia social, e a experiência com a Internet com o processo de adoção da compra pela internet. Foi elaborado um modelo integrativo que possibilitasse explicar a relação entre esses fatores e a compra pela internet, e foi desenvolvida uma pesquisa de campo considerando uma amostra não probabilísti- ca de estudantes. Foi utilizado o método multivariado de modelagem de equações estruturais, aplicado por meio da técnica Partial Least Squares (PLS) para a verificação, explicação e comparação das relações entre os construtos. Os resultados mostram que a intenção da compra pela internet é diretamente influenciada pela atitude e pela inovatividade, e a atitude é influenciada pelo envolvimento. Não foi encontrada relação entre a experiência com a internet e a compra pela internet.

PALAVRAS-ChAVE Comportamento do consumidor, internet, inovatividade, envolvimento, atitude.

Abrão Caro abraocaro@mackenzie.br

Professor do Centro de Ciências Sociais e Aplicadas, Universidade Presbiteriana Mackenzie - São Paulo - SP, Brasil

José Afonso Mazzon jamazzon@usp.br

Professor da Faculdade de Economia, Administração e Contabilidade da Universidade de São Paulo - São Paulo - SP, Brasil

Barbara Caemmerer barbara.caemmerer@essca.fr

Professora da School of Management, Ecole Supérieure des Sciences Commerciales D'angers e L'Université Nantes Angers Le Mans, France

Matthias Wessling mail@wessling.info

Professor da School of Management, RWTH Aachen University, Netherlands

Abstract This work seeks to analyze the inter-relationships that exist between innovation, involvement and attitude in the decomposed model of the Theory of Planned Behavior (TPB), as applied in social psychology, and experience of using the Internet in the process of buying on-line. An integrative model was prepared that made it possible to explain the relationship that exists between these factors and purchasing from the Internet. A field survey was undertaken with a non-probabilistic sample of students. A multivariate structural equation modeling method was used and applied by the Partial Least Squares (PLS) technique for checking, explaining and comparing the relationship between the constructs. The results show that intention to buy via the Internet is directly influenced by attitude and innovativeness, and attitude is influenced by involvement. No relationship was found between experience in using the Internet and buying from it.

keywords Consumer Behavior, Internet, innovativeness, involvement, attitude.

Resumen Este trabajo se propone analizar las interrelaciones entre la innovación, la participación, la actitud dentro del modelo Theory of Planned Behavior (TPB) descompuesto desarrollado en la psicología social, y la experiencia con la Internet con el proceso de adopción de la compra por la Internet. Se elaboró un modelo integrativo que hiciese posible explicar la relación entre eses factores y la compra por la Internet, y se desarrolló una investigación de campo considerando una muestra no probabilística de estudiantes. Fue utilizado el método multivariado de modelaje de ecuaciones estructurales, aplicado por medio de la técnica Partial Least Squares (PLS) para la verificación, explicación y comparación de las relaciones entre los constructos. Los resultados muestran que la intención de la compra por la Internet es directamente influenciada por la actitud y por la innovación, y la actitud es influenciada por la participación. No se encontró relación entre la experiencia con la Internet y la compra por la Internet.

Palabras clave Comportamiento del consumidor, Internet, innovación, participación, actitud. 


\section{INTRODUÇÃO}

A internet tem provocado grandes alterações na forma como as pessoas se comunicam, trabalham, conduzem pesquisas e se informam, e está começando a alterar a forma como elas compram.

De acordo com Aun (2007), enquanto a população mundial cresce em uma média de $1,1 \%$ ao ano, a população de internautas cresce em uma média de $6,6 \%$ ao ano. Segundo o mesmo autor, o maior crescimento na população de internautas ocorrerá no Brasil, na Rússia, Índia e China. Estatísticas realizadas por Internet Worldstats (2011) mostram que o número de internautas chegou a $30,2 \%$ da população mundial em março de 2011 e, no Brasil, nesse mesmo período, a penetração da internet alcançou $37,4 \%$ da população brasileira.

O comércio pela internet, embora em volumes baixos em relação às compras nas lojas físicas, tem crescido continuamente a taxas muito elevadas, o que poderá mudar diretamente a maneira como as empresas e os consumidores se relacionam. De acordo com dados do U.S. Census Bureau News (2011), as vendas ao consumidor nos Estados Unidos no período entre 2004 e 2007 apresentaram um crescimento médio anual de $5,2 \%$, enquanto as vendas pela internet, nesse mesmo período, tiveram um crescimento médio anual de $24,1 \%$. No período recessivo, as vendas totais do varejo caíram 1,3\% em 2008, em relação a 2007, e também tiveram queda de $8,0 \%$ em 2009 , em relação ao ano anterior, enquanto as vendas pela internet apresentaram crescimentos de 3,0\% e 2,1\% nesses mesmos períodos. Em 2010, o crescimento anual no total do varejo nos Estados Unidos foi de 6,9\%, e os dados do primeiro trimestre de 2011 mostram um crescimento de 8,6\% sobre o mesmo período de 2010 , enquanto as vendas pela internet cresceram $15,2 \%$ e $17,6 \%$ respectivamente.

O consumidor passou a ter novas opções e formas para comprar. Uma das áreas de pesquisa à qual estudiosos têm se dedicado é a investigação dos fatores que apresentariam uma relação de causa e efeito ou que estariam relacionados à compra pela internet. A investigação desses fatores justifica-se pelo impulso que esse conhecimento poderá acarretar ao processo de disseminação do uso da internet para o comércio, conjugado com os seus benefícios. Esse enfoque é o fator motivador, direcionador e delimitador do presente trabalho.

Foram selecionados como fatores influenciadores para o presente estudo:
- A inovatividade, que é o grau de receptividade a novos produtos, serviços ou práticas. A inovatividade foi escolhida por se apresentar como preditora da compra on-line ou de inovações em estudos anteriores, como Blake, Neuendore e Valdiserri (2003), Goldsmith (2001), Joseph e Vyas (1984), Mahajan, Muller e Bass (1990), Phau e Lo (2004), Im, Bayus e Mason (2003), Cittrin e outros (2000).

- O envolvimento, que é o nível de importância pessoal ou interesse por um produto, serviço ou prática. Pelo mesmo motivo, foi escolhido o envolvimento baseado nos estudos de Hawkins, Mothersbaugh e Best (2007), Wu (2002), Zaichkowsky(1986) e Blackwell, Miniard e Engel (2005).

- A atitude como fator influenciador da compra on-line foi escolhida por estar relacionada à predisposição em relação à ação, e muitos autores estudaram sua ligação com a adoção de inovações, como Karahanna, Straub e Chervany (1999), Gefen, Karahanna e Straub (2003), Davis (1989), Venkatesh e outros (2003), Mathieson, Peacock e Chin (2001), Taylor e Todd (1995) e Shih e Fang (2004). Shih e Fang (2004) utilizaram os modelos TPB puro e TPB decomposto para estudar o comportamento do usuário de internet em bancos e concluíram que o modelo decomposto melhora o entendimento das relações entre a atitude e o comportamento, o que levou à escolha do modelo decomposto da Teoria da Ação Planejada (TPB).

- A experiência com a internet, por ser um poderoso influenciador das atitudes em relação à web, de acordo com Limeira (2007).

Procura-se, neste trabalho, o levantamento de informações sobre o consumidor on-line e a análise dessas informações utilizando técnicas adequadas. Busca-se um conhecimento maior sobre as práticas de compras usando a tecnologia da internet pelos indivíduos e as características e os motivos que os levam a essa escolha.

Esse é o contexto em que se apresenta a proposta deste trabalho, que procurará responder: Qual é a influência da inovatividade, do envolvimento, da atitude e da experiência com a internet na adoção da compra pela internet?

O objetivo específico deste trabalho é analisar as relações entre os fatores inovatividade, envolvimento, atitude e experiência com a internet, que afetam a decisão de adoção da compra pela internet. 


\section{FUNDAMENTAÇÃO TEÓRICA}

O comércio eletrônico ou e-commerce é basicamente a realização de transações empresariais via redes de telecomunicações, especialmente a internet (TURBAN, MCLEAN, WETHERBE, 2004). Entre os vários modelos de comércio eletrônico, o varejo on-line é a venda direta B2C em que lojas virtuais são projetadas, em geral, em formato de catálogo eletrônico. O consumidor pode comprar de sua residência, a qualquer hora do dia, nos sete dias da semana.

A internet trouxe muitas vantagens aos consumidores. Conforme Turban, McLean e Wetherbe (2004), os principais benefícios do comércio eletrônico para os consumidores são produtos e serviços mais baratos, possibilitados pela facilidade de comparação de preços de mercado; escolhas mais diversificadas, por passarem a ter acesso a mais fornecedores; comodidade de horário, em que o acesso pode ser efetuado a qualquer hora do dia; informações detalhadas dos produtos rapidamente; acesso a produtos personalizados; participação em leilões virtuais; interação com outros consumidores para trocar e compartilhar ideias.

Apesar de todo o crescimento apresentado, o comércio eletrônico está apenas iniciando. As diferenças entre as lojas virtuais e as lojas físicas, além das estratégias inovadoras de marketing nesse novo ambiente, devem estar mudando hábitos e estilos de compra. Não obstante as novas opções e formas para comprar que o consumidor passou a ter, muito pouco se sabe sobre as mudanças em relação às suas atitudes, percepções e fatores motivadores da adoção ou não da compra virtual. O futuro do e-commerce também depende de se os usuários atuais da internet vinculados apenas em comunicação, entretenimento e pesquisa passarão a comprar on-line.

A aceitação de um produto novo, de um novo serviço ou de uma inovação como a compra pela internet torna necessário entender a razão e a forma pela qual os consumidores realizam suas compras. É preciso conhecer as atividades diretamente envolvidas em obter, consumir e dispor de produtos e serviços incluindo os processos decisórios que antecedem e sucedem essas ações; para tal, é necessário estudar o comportamento do consumidor, objeto de análise do tópico seguinte.

\section{Comportamento do consumidor}

O estudo do comportamento do consumidor é uma disciplina recente. As bases dessa nova disciplina de marketing foram formadas utilizando-se vários conceitos emprestados de outras disciplinas, como psicologia (estudo de indivíduos), sociologia (estudo de grupos), psicologia social (o estudo de como um indivíduo age quando em grupo), economia (o estudo dos padrões de consumo na sociedade) (SMITH e RUPP, 2003) e antropologia (estudo do homem, seus costumes, crenças e hábitos). O consumidor sofre influências de fatores culturais, sociais, individuais e psicológicos. Também é estimulado pelo ambiente externo em que se situa, que são fatores de âmbito econômico, tecnológico, político e cultural, além de estímulos do composto de marketing: produto, por meio de seu conjunto de atributos e de vantagens procuradas; preço, representado como custo de aquisição e utilidade; distribuição, como disponibilidade no tempo e no espaço; propaganda e promoção, com informação e persuasão como fator incitante à aquisição, que em seguida passa por um processo decisório que leva à escolha do produto, da marca, do revendedor, do momento da compra e da quantidade comprada.

Entre os fatores influenciadores do comportamento do consumidor, foram selecionados os fatores atitude, inovatividade, envolvimento e experiência com a internet, assuntos abordados nos próximos subtópicos.

\section{Atitude}

A atitude de uma pessoa é uma predisposição para com uma conduta, é a avaliação favorável ou desfavorável que a pessoa faz de seu comportamento, ela precede e produz o seu comportamento. Na visão contemporânea, as atitudes são formadas por meio de crenças e de sentimentos sobre o objeto da atitude. A identificação da maneira como as atitudes são formadas é importante porque fornece informações para aqueles que querem influenciar as atitudes do consumidor. Um dos principais modelos conceituais sobre o tema é a Teoria da Ação Racional (TRA) (VENKATESH e outros, 2003).

Ajzen e Fishbein elaboraram a TRA, em que o antecedente mais diretamente ligado a um comportamento é a intenção que o indivíduo tem de realizá-lo (AJZEN, 1985). A intenção é a predisposição para executar um determinado comportamento. Essa intenção depende de dois fatores, sendo um de natureza pessoal, que é a atitude para com o comportamento, que depende da avaliação pela pessoa de maneira positiva ou negativa das consequências desse comportamento, e o segundo fator é a norma subjetiva, que considera a intensida- 
de da pressão social que a pessoa sente em relação a determinado comportamento. Segundo os autores, o modelo TRA foi desenvolvido para lidar com o comportamento como um processo racional de formação de intenções e, nesse contexto, tem mostrado bons resultados. Entretanto, não são todas as intenções formadas por um processo racional que conseguem levar a um comportamento desejado; deficiências pessoais e obstáculos externos podem interferir na consecução de determinado comportamento. E, nesse caso, complicações são encontradas na aplicação do modelo TRA. Isso levou os autores a desenvolver um novo modelo, a TPB. Essa nova proposta teórica considera que a intenção de realizar determinado comportamento é resultado não só da avaliação que o indivíduo faz desse comportamento e da pressão social para exercê-lo, mas de mais um elemento, que é o controle comportamental percebido, ou seja, é o grau de facilidade ou dificuldade que o indivíduo acredita ter para executar determinado comportamento, reflete experiências passadas e antecipa impedimentos e obstáculos.

Bobbitt e Dabholkar (2001) propuseram um amplo modelo integrativo com o propósito de entender e prever a adoção da internet utilizando o modelo TPB de Ajzen e Fishbein. Consideraram desde fatores situacionais, fatores associados com a categoria do produto, o controle percebido, resultados de tentativas anteriores, assim como riscos associados ao uso da internet. Os mesmos autores não utilizaram as normas subjetivas como possíveis influenciadores da atitude em relação à compra pela internet em seu modelo, considerando que seu efeito seria desprezível.

Taylor e Todd (1995) formularam a teoria TPB decomposta agregando a teoria da difusão da inovação de Rogers à TPB de Ajzen e Fishbein. Segundo essa teoria, as crenças comportamentais influenciam a intenção e ficaram subdivididas em:

- Vantagem relativa, que se refere ao grau com que uma inovação traz benefícios adicionais e está positivamente relacionada com o nível de adoção de uma inovação.

- Compatibilidade, que é o nível em que a inovação se combina com os valores e responsabilidades de uma pessoa.

- Complexidade, que representa o grau com que uma inovação é percebida como difícil de entender, aprender ou operar.

- Segundo estudos de Taylor e Todd (1995) e Moore e Bensabat (1991), vantagem relativa é o construto com mais influência nas crenças comportamentais.
- As crenças de controle ficaram subdivididas em:

- Eficácia, que é a habilidade de conseguir efetuar uma operação com sucesso;

- Condições facilitadoras, que refletem a disponibilidade dos recursos necessários para determinado comportamento. As condições facilitadoras são relacionadas a fatores como tempo, dinheiro e infraestrutura.

Na figura 1, abaixo, é apresentado o modelo da TPB decomposta.

Taylor e Todd (1995) concluíram que tanto o modelo da teoria TRA quanto o da teoria TPB são válidos quando utilizados para análises de fenômenos, porém a teoria TPB decomposta tem um melhor poder explicativo que o TPB puro na adoção de uma inovação, porque fornece detalhes específicos que podem auxiliar na compreensão do fenômeno, o que foi confirmado por Shih e Fang (2004).

\section{0 processo de adoção e difusão da inovatividade}

Quando um novo produto ou uma nova tecnologia é introduzido no mercado, os consumidores tomam consciência e aprendem a seu respeito, decidem se vão comprar, quando comprar e se vão repetir a compra no futuro. Conforme Engel, Blackwell e Miniard (2000, p. 579), inovatividade é o grau em que um indivíduo adota uma inovação relativamente mais cedo do que os outros.

Existem duas linhas de pesquisa em relação à adoção de uma inovação: a predisposição à inovação e a inovatividade limitada a um domínio ou produto.

Para Joseph e Vyas (1984), a primeira linha de pesquisa enfoca o estilo cognitivo, que incorpora características intelectuais, perceptuais e atitudinais. Inovatividade, nesse sentido, é o grau com que um indivíduo é receptivo a novas ideias e toma decisões sobre inovações, independentemente de influência de outros. Entretanto, Citrin e outros (2000) não encontraram relação entre os inovadores abertos a novidades e a adoção da compra pela internet.

Uma outra linha de pesquisa enfoca a inovatividade limitada a um domínio ou a um produto específico, assim procura refletir a tendência a se adotar uma inovação dentro de uma área de interesse. Nessa linha, Goldsmith e Hofacker (1991) desenvolveram a escala Domain Specific Innovativeness (DSI) para medir esse conceito. Goldsmith (2001) aplicou a escala DSI 
e confirmou que a inovatividade está associada positivamente com o número de horas de uso da internet e com a compra on-line. Cittrin e outros (2000) utilizaram a mesma escala DSI e concluíram que inovatividade é positivamente associada com volume de compra on-line com base em estudo sobre hábitos com estudantes universitários. Alcañiz e outros (2008) e Manzano e outros (2009) estudaram e associaram a inovatividade à compra on-line.

O modelo de adoção amplamente usado é o apresentado em 1962 por Rogers (2003), que demonstrou que a distribuição dos adotantes de uma inovação em relação ao tempo aproxima-se de uma curva normal. A distribuição normal de frequência possui características que são úteis para classificar os adotantes, como a média e o desvio-padrão. A média e o desvio-padrão foram utilizados para dividir a distribuição normal adotada em cinco categorias de consumidores, separadas por linhas verticais, conforme a Figura 2. A área à esquerda da média menos dois desvios-padrões inclui 2,5\% dos adotantes: são os inovadores. Os 13,5\% seguintes são chamados de adotantes imediatos. Os próximos 34\% são a maioria dos adotantes imediatos. À direita da média e entre a média e um desvio-padrão, estão os $34 \%$ que representam os adotantes tardios, e os $16 \%$ seguintes são os retardatários.

Para Blake, Neuendore e Valdiserri (2003), a inovatividade pode ser o fator fundamental determinante da qualidade e da quantidade da compra pela internet. Muitos trabalhos utilizaram a inovatividade: Eastlick e Lotz (1999) concluíram, com base em pesquisa realizada com 2.500 usuários de TV a cabo nos Estados Unidos, que a inovatividade é forte preditora de inovadores e também de não adotantes. Alcañiz e outros (2008) mostraram que a inovatividade exerce uma relação positiva em relação à intenção de compra pela internet. Manzano e outros (2009) concluíram em estudo que a inovatividade é um construto-chave na adoção da internet para a adoção de produtos bancários e desempenha um papel importante na redução da percepção de risco no uso da internet.

\section{Envolvimento}

Conforme Zaichkowsky (1986), o envolvimento é definido como a relevância do objeto percebida por uma pessoa com base em suas necessidades, valores e interesses. Assim, envolvimento está relacionado com a importância ou o interesse de um indivíduo por um objeto, produto ou situação. À medida que o envolvimento com um produto aumenta, o consumidor dedica mais atenção aos anúncios relacionados ao produto. Para Zaichkowsky (1986), um indivíduo, quando está envolvido, presta mais atenção, dá mais importância e se comporta de uma forma diferente de quando não está. Ainda segundo a mesma auto-

\section{Figura 1 - Modelo TPB decomposta}

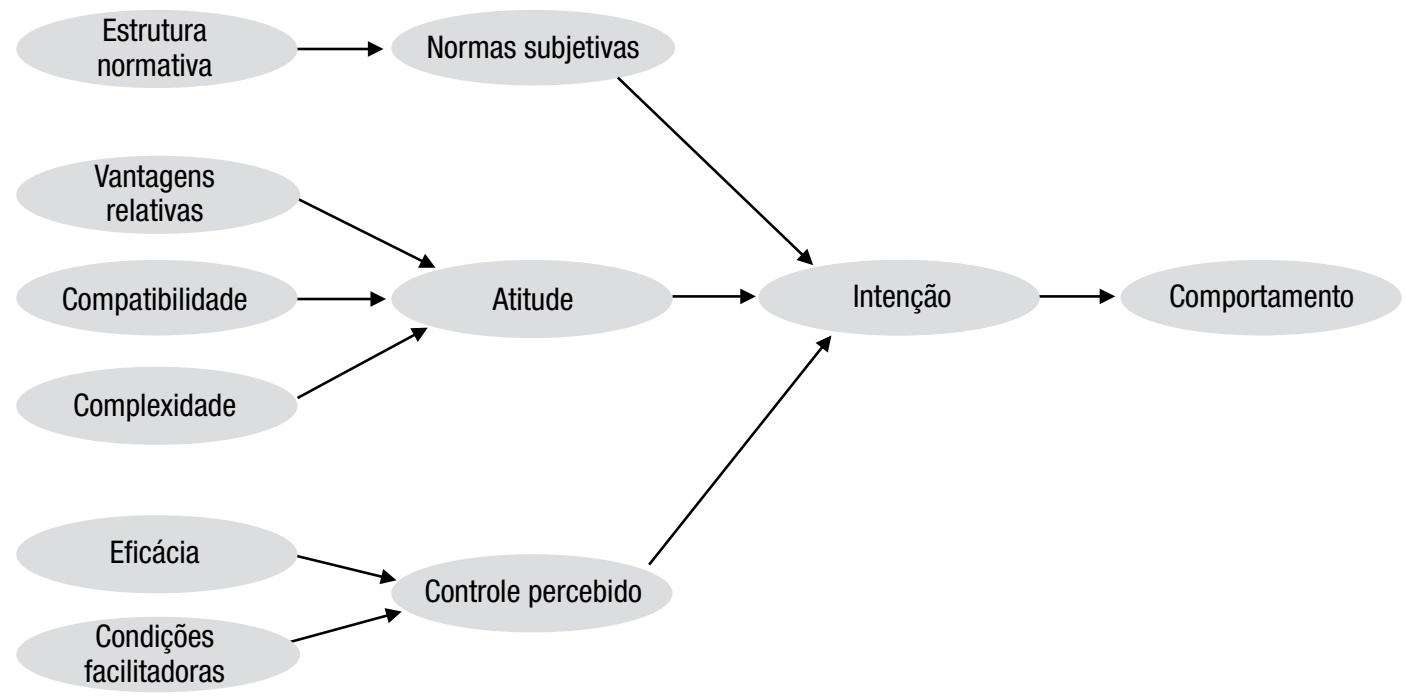


ra, os antecedentes do envolvimento podem ser caracterizados por três fatores: o sistema de valores da pessoa, que é individual e determina se uma pessoa está envolvida com determinado objeto; o segundo fator está relacionado com o estímulo que a pessoa recebe, por meio do conteúdo de uma mensagem ou da fonte da mensagem; e o terceiro fator é a situação dada pela ocasião da compra ou do uso do objeto.

A medida do envolvimento é importante para muitas aplicações de marketing. Uma abordagem de medida por níveis de envolvimento permite captar a diversidade da construção do envolvimento. Uma das medidas mais usadas do estado de envolvimento é a escala de Zaichkowsky. Essa escala é bipolar Personal Involvement Inventory (PII) e foi formulada com 20 itens, e depois revisada e reformulada reduzindo os itens para 10 (ZAICHKOWSKY, 1994). Bienstock e Stafford (2006), McQuarrie e Munson (1987), entre outros, testaram e validaram a escala.

Vários estudos analisaram o envolvimento e sua influência na compra pela internet. Wu (2002) desenvolveu uma pesquisa procurando a influência dos fatores como características demográficas do consumidor, seu estilo de vida, suas necessidades percebidas e a situação em relação ao envolvimento e suas consequências na compra on-line. Ele concluiu que o nível de envolvimento do consumidor com uma categoria de produto ou serviço é o principal determinante de sua compra ou uso.

Golsmith e Flynn (2004) concluíram, em estudo utilizando técnicas de análise multivariadas, que variá- veis demográficas como idade, sexo e renda possuem menor poder de explicação na compra de roupas on-line. Inovatividade em moda é fracamente relacionada com compra on-line, mas o contrário acontece com envolvimento com moda. Para Hawkins, Mothersbaugh e Best (2007), o envolvimento influencia inúmeros comportamentos ao consumidor e é um determinante importante de como os consumidores formam atitudes e tomam decisões de compra.

\section{Experiência com a internet}

A navegação por diversos sites da internet forma a experiência com a internet, e é o primeiro passo para a adoção da compra on-line (HUANG, 2003).

Embora a experiência on-line não garanta a compra pela internet, para Novak, Hoffman e Yung (2000), a experiência com a internet pode ajudar a determinar se uma compra on-line será realizada. Muitas das compras on-line são iniciadas, porém não se concretizam (CHO, 2004).

Vários autores apontaram a relação positiva entre a experiência com a internet e a adoção da compra on-line. Limeira (2007), baseada em pesquisa realizada por Hammond, McWilliam e Diaz em 1998, afirmou que a experiência de uso da internet é um poderoso influenciador das atitudes em relação à web. Segundo Johnson e outros (2004), a experiência com a internet motiva os consumidores para a compra on-line, o que é confirmado por estudos de Xia (2002).

Para Wolfinbarger e Gilly (2001), a motivação para

\section{Figura 2 - Tempo de adoção de inovações}

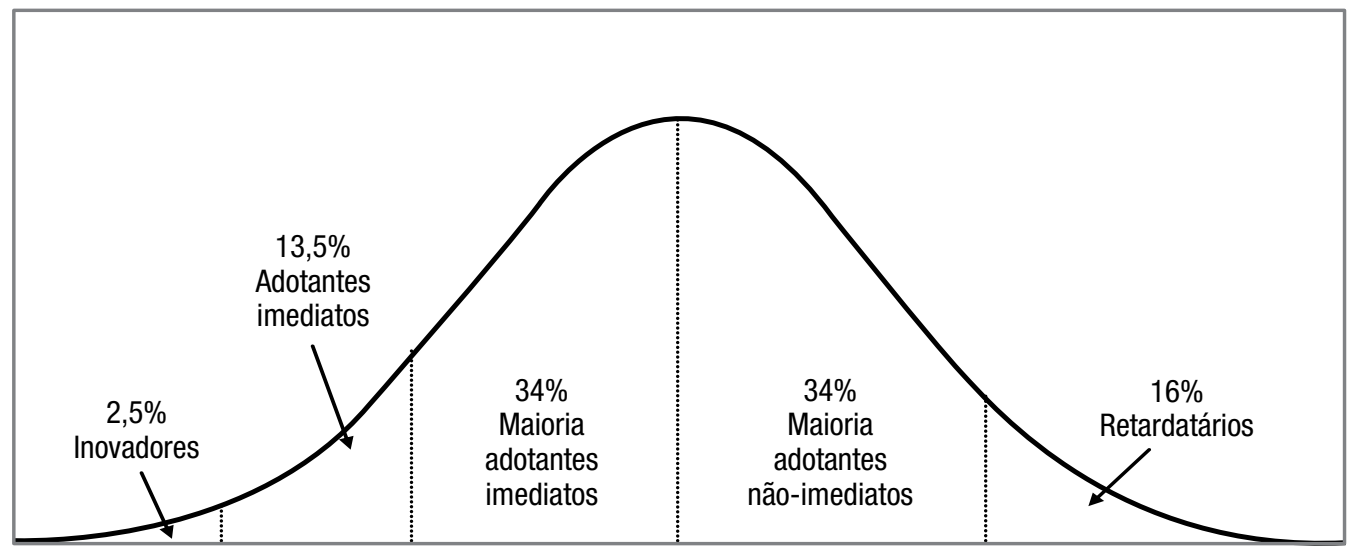

Fonte: ROGERS (2003) 
comprar on-line é um fator que pode influenciar a passagem de experiência com a internet para a compra on-line. Nessa mesma direção, de acordo com Korzaan (2003), o processamento e a integração de informações proporcionado pelas páginas dos sites da internet pode influenciar a mudança de atitude. O mesmo autor analisou a relação entre a experiência on-line e a adoção da compra pela internet e concluiu que a experiência on-line forma uma atitude mais favorável e positiva com a compra on-line.

\section{METODOLOGIA DA PESQUISA}

O modelo conceitual, apresentado na Figura 3 abaixo, tem o objetivo de explicitar as relações entre os conceitos que foram utilizados na pesquisa.

A variável adoção da compra pela internet é a variável dependente, as variáveis inovatividade, experiência com a internet e envolvimento são variáveis independentes, a variável atitude é dependente e independente.

O levantamento dos dados foi efetuado por autopreenchimento pela internet em questionário cadastrado em site especialmente desenvolvido para esta pesquisa, contendo as instruções para seu preenchimento e precedido de informações sobre os objetivos da pesquisa e o uso de seus resultados exclusivamente para fins acadêmicos. Os respondentes foram convidados a participarem da pesquisa voluntariamente.

O instrumento de coleta dos dados foi organizado contendo quatro questões sobre as características dos respondentes e 89 questões referentes aos construtos do modelo conceitual medidas por meio das escalas e fontes que estão relacionadas na Tabela 1 abaixo.

Como o questionário elaborado é longo, contendo 93 questões, o seu preenchimento poderia se tornar cansativo e ocorreria um problema com a atenção dos respondentes nas respostas às últimas questões, prejudicando-as. Diante desse possível problema, adotou-se

\section{Figura 3 - Modelo conceitual}

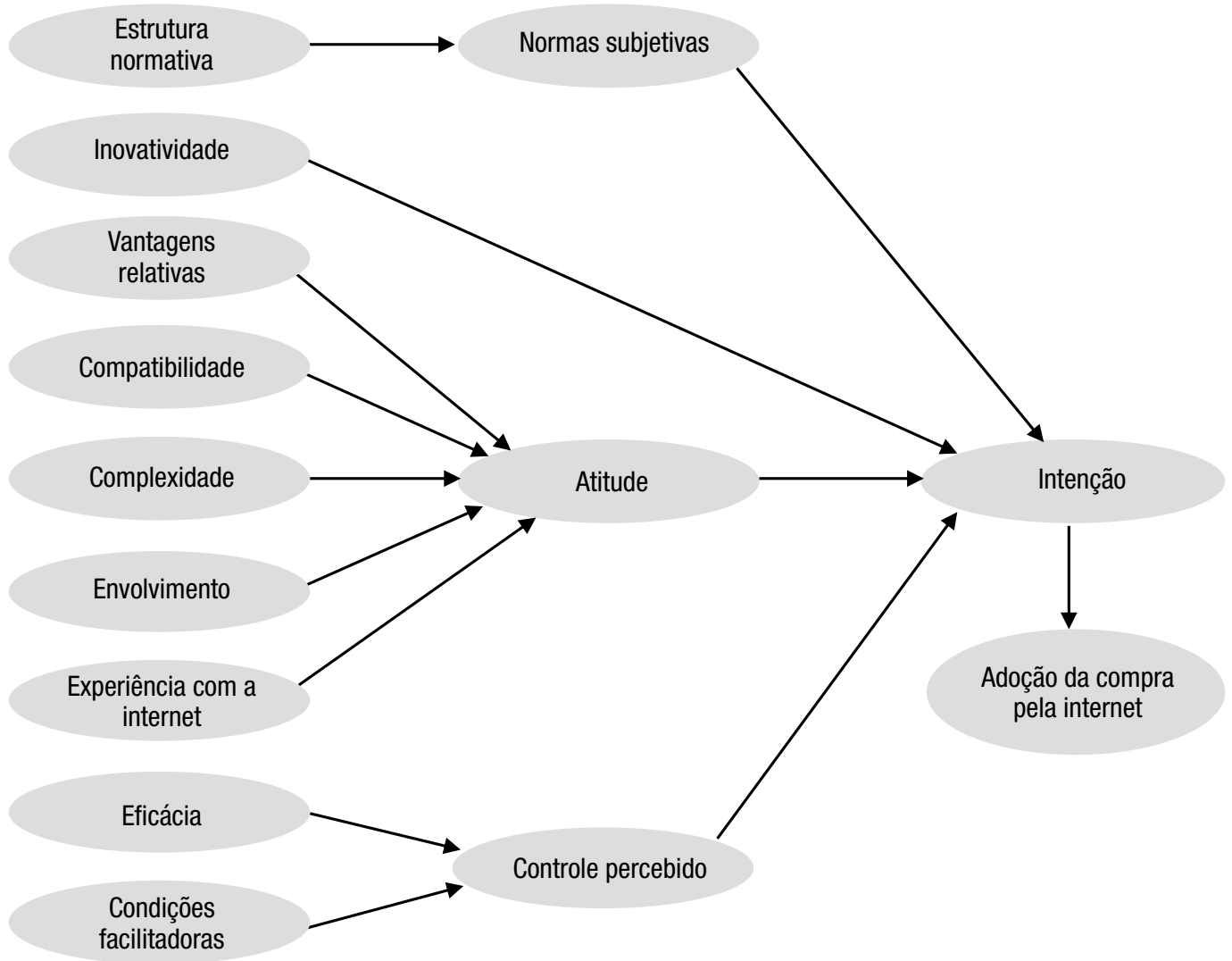




\section{Tabela 1 - Estrutura do instrumento de coleta}

\begin{tabular}{|c|c|c|c|c|}
\hline \multicolumn{2}{|c|}{ Construto } & $\begin{array}{l}\text { Número de } \\
\text { questões }\end{array}$ & Escala & Fonte \\
\hline \multicolumn{2}{|c|}{ Inovatividade } & 6 & $\begin{array}{l}\text { Domain Specific Innovativeness } \\
\text { DSI de Goldsmith e Hofaker }\end{array}$ & Bearden e Netemeyer (1999) \\
\hline \multirow{10}{*}{ 吾 } & Estrutura normativa & 8 & & Taylor e Todd (1995) \\
\hline & Normas subjetivas & 4 & & Taylor e Todd (1995) \\
\hline & Vantagens relativas & 8 & & Taylor e Todd (1995) e Moore e Bensabat (1991) \\
\hline & Compatibilidade & 8 & & Taylor e Todd (1995) e Moore e Bensabat (1991) \\
\hline & Complexidade & 8 & & Taylor e Todd (1995) e Moore e Bensabat (1991) \\
\hline & Eficácia & 8 & & Taylor e Todd (1995) \\
\hline & Condições facilitadoras & 8 & & Taylor e Todd (1995) \\
\hline & Controle percebido & 4 & & Shih e Fang (2004) \\
\hline & Atitude & 4 & & Taylor e Todd (1995) \\
\hline & Intenção & 4 & & Taylor e Todd (1995) e Shih e Fang (2004) \\
\hline \multicolumn{2}{|c|}{ Envolvimento } & 10 & $\begin{array}{l}\text { Personal Involvement Inventory } \\
\text { PII de Zaichkowsy }\end{array}$ & Bearden e Netemeyer (1999) \\
\hline \multicolumn{2}{|c|}{ Experiência com a internet } & 3 & & Novak entre outros (2000) \\
\hline \multicolumn{2}{|c|}{ Adoção da compra pela internet } & 6 & & Hernandez e Mazzon (2007) \\
\hline
\end{tabular}

a randomização na apresentação das perguntas aos respondentes, de modo que houvesse uma rotatividade das questões e cada respondente recebesse o questionário com as questões em ordem diferente. Esse método faz com que, se houver menor atenção no preenchimento das últimas questões, esse problema ficaria diluído entre todas as questões.

Também foram tomados cuidados para evitar respostas repetidas pelo mesmo respondente, por meio da identificação do equipamento de acesso ao site.

Foi desenvolvido um programa específico para o procedimento de coleta contendo as questões e a randomização das perguntas, que foi cadastrado em um site.

Foi efetuado um pré-teste com 30 respondentes e foram corrigidos os problemas de clareza e precisão dos termos, adequação da quantidade de perguntas, entendimento dos objetivos da pesquisa e aperfeiçoamento da funcionalidade do questionário e teste das escalas, antes de ser estendido à amostra, além da medida do tempo necessário para seu preenchimento. Não houve desistências após iniciado o preenchimento do questionário.

Foram contatados professores de universidades para que divulgassem essa pesquisa aos seus alunos. O número de respondentes foi elevado, chegando a 519, sendo que 474 compraram pela internet pelo menos uma vez, sem distinção de valor ou produto, e 45 não compraram. Como o questionário foi extenso, houve a ocorrência de missing values ou dados não preenchidos. Os dados não preenchidos, entre os que compraram pela internet, foram em pequeno número, sendo a média entre todas as questões igual a $0,8 \%$ e o máximo igual a $2,1 \%$.

Foi formada uma matriz com as respostas dos que compraram pela internet contendo um indicador para cada questão, as questões contendo escores reversos tiveram seu valor subtraído de 11 e as questões referentes aos construtos referentes à atitude foram formadas pelo produto entre os pares de questões correspondentes.

Os missing values foram substituídos pela média dos casos preenchidos pelos respondentes para cada questão, exceto as questões dos subgrupos compostos pelo produto de pares de questões, seu produto foi substituído pela média dos produtos das questões correspondentes em que ambas foram preenchidas pelos respondentes.

Os dados coletados foram analisados por meio da técnica de Modelagem de Equações Estruturais que, de acordo com Hair e outros (1998), possibilita esti- 
mar muitas equações ao mesmo tempo, e elas podem ser inter-relacionadas, ou seja, a variável dependente em uma equação pode ser independente em outra(s), possibilitando modelar relações complexas.

Alguns programas têm sido utilizados pelos pesquisadores para a modelagem de equações estruturais, como LISREL, AMOS e PLS, entre outros. PLS é uma técnica de modelagem de equações estruturais baseada em um método de regressão que maximiza a variância explicada (HAIR e outros, 2009).

Henseler, Ringleand e Sinkovics (2009), em pesquisa de artigos sobre marketing realizada nas principais revistas acadêmicas, constataram que PLS teve uma participação crescente em trabalhos acadêmicos, principalmente na área de marketing internacional, e sua expectativa é de uma maior utilização neste novo milênio. Ainda segundo os mesmos autores, a popularidade do PLS entre os pesquisadores é devida às seguintes características:

- A possibilidade de considerar modelos de mensuração reflexivos e formativos.

- Pode ser usado para estimar modelos com amostras de pequeno tamanho.

- PLS é metodologicamente vantajoso em relação às outras técnicas quando não há convergência nos resultados e, além disso, consegue lidar com modelos complexos em que o número de variáveis latentes e indicadores seja grande em relação ao número de observações.

- Não há exigência de normalidade nas distribuições dos dados.

Além dessas vantagens, Hair e outros (2009) afirmam que PLS pode lidar com todos os tipos de dados, tanto numéricos como não numéricos, e com até um único indicador por construto. Chin (1998), por sua vez, destaca que uma das razões do crescimento da atenção ao PLS pelos pesquisadores deve-se ao fato de que ele é consistente com os preceitos da modelagem de equações estruturais e provê com habilidade previsões e entendimento dos papéis e da formação de cada construto, além da relação entre os construtos.

\section{ANÁLISE DOS DADOS COLETADOS}

Em relação ao gênero, a amostra apresentou uma participação um pouco maior de representantes do gênero feminino do que representantes do gênero masculino $(52,3 \%$ e $47,7 \%$ respectivamente). A faixa etária dos respondentes da amostra concentrou-se em um público mais jovem, com quase 94\% com idade até 34 anos, e isso se deveu à forma como foi coletada a amostra (por intermédio de universidades), e teve uma grande participação de respondentes com elevada escolaridade, com $72 \%$ de graduados ou em curso superior e $25 \%$ de respondentes cursando ou tendo concluído a pós-graduação.

Os dados levantados foram analisados por meio da técnica estatística multivariada de modelagem de equações estruturais aplicadas por meio do software Smart PLS 2.0, com base na amostra total de 474 casos de respondentes que compraram pela internet; os resultados obtidos são apresentados na Tabela 2 a seguir.

\section{Avaliação do modelo de mensuração}

As questões coletadas dos respondentes, chamadas de indicadores no modelo de mensuração, são utilizadas para medir os construtos ou variável latente. Desse modo, o construto inovatividade tem seis indicadores, o construto envolvimento, 10, e assim por diante. A mensuração de cada construto foi avaliada em relação aos seus indicadores. Foram utilizados os seguintes critérios para a avaliação do modelo de mensuração, conforme recomendado por Henseler, Ringleand e Sinkovics (2009) e Chin (1998): confiabilidade composta, AVE, confiabilidade dos indicadores e validade discriminante pelos critérios Fornell-Larcker e Cross-loadings.

- Confiabilidade composta: é uma medida de consistência interna considerada mais apropriada para diferentes medidas que o Alpha de Cronbach, porque leva em consideração que os indicadores possuem diferentes cargas. Nenhum dos construtos obteve índice abaixo de 0,6. O índice mais baixo foi o da variável latente $\operatorname{Exp} 0,616$.

- Average Variance Extracted (AVE) - variância extraída média: é considerado um critério de validade convergente. Um valor de AVE de, no mínimo, 0,5 indica validade convergente suficiente, mostrando que a variável latente explica mais da metade da variância que a média de seus indicadores. Os resultados apresentados na Tabela 2 indicam que as variáveis latentes que não chegaram ao nível mínimo de 0,5 foram Adoção da Compra pela Internet, 


\section{Tabela 2 - Modelo geral 1}

\begin{tabular}{|c|c|c|c|c|}
\hline Construto & Descrição do construto & AVE & $\begin{array}{c}\text { Raiz quadrada } \\
\text { de AVE }\end{array}$ & $\begin{array}{c}\text { Confiabilidade } \\
\text { composta }\end{array}$ \\
\hline Ad_Compra & Adoção da compra pela internet & 0,428 & 0,655 & 0,782 \\
\hline Atitude & Atitude & 0,752 & 0,867 & 0,924 \\
\hline Compatib & Compatibilidade & 0,742 & 0,861 & 0,920 \\
\hline Complex & Complexidade & 0,410 & 0,640 & 0,703 \\
\hline Cond_Fac & Condições facilitadoras & 0,540 & 0,735 & 0,824 \\
\hline Contr_Perc & Controle percebido & 0,665 & 0,815 & 0,888 \\
\hline Eficac & Eficácia & 0,625 & 0,790 & 0,869 \\
\hline Envolv & Envolvimento & 0,484 & 0,696 & 0,895 \\
\hline Estr_Norm & Estrutura normativa & 0,670 & 0,818 & 0,890 \\
\hline Exp & Experiência com a internet & 0,391 & 0,625 & 0,616 \\
\hline Inov & Inovatividade & 0,410 & 0,640 & 0,795 \\
\hline Inten & Intenção & 0,600 & 0,775 & 0,853 \\
\hline Norm_Subj & Normas subjetivas & 0,768 & 0,876 & 0,930 \\
\hline Vant_Rel & Vantagens relativas & 0,650 & 0,806 & 0,881 \\
\hline
\end{tabular}

Complexidade, Envolvimento, Experiência com a Internet e Inovatividade.

- Confiabilidade dos indicadores: conforme Chin (1998), o limite mínimo para a relação entre o indicador e a variável latente é 0,5 . Um indicador de cada um dos construtos inovatividade, envolvimento e adoção da compra pela internet e dois indicadores do construto Ccmplexidade apresentaram relação abaixo desse nível.

- Critério Fornell-Larcker: para avaliar a validade discriminante, cada variável latente deve partilhar a variância mais com seu bloco de indicadores do que com qualquer indicador de outra variável latente, assim a raiz quadrada do AVE de cada variável latente deve ser maior que a correlação com as outras variáveis latentes.

A Tabela 3 acima mostra, na diagonal, a raiz quadrada do AVE, e nos demais campos a correlação entre as variáveis latentes. A raiz quadrada do AVE de cada variável latente é maior que a correlação com as outras variáveis latentes, exceto nos seguintes casos das variáveis Inten e Eficac.

Cross-loadings: se um indicador possuir uma correlação maior com uma variável latente do que com a sua respectiva, o modelo tem de ser revisto. É mais uma medida de validade discriminante. A análise Cross-loadings indicou problemas somente em um dos indicadores da variável envolvimento.

Com base nas avaliações feitas, foram retirados os indicadores com baixa confiabilidade e do critério Cross-Loadings. O modelo foi reprocessado e foi efetuada uma segunda análise do modelo de mensuração com os novos parâmetros. Os resultados são apresentados na Tabela 4 seguinte.

$\mathrm{Na}$ análise da confiabilidade composta, nenhum dos construtos obteve índice abaixo de 0,6. O índice mais baixo foi o da variável latente Exp 0,747.

Os resultados apresentados na tabela 4 indicam que as variáveis latentes superaram o nível mínimo do AVE de 0,5, exceto a variável latente Inov, com índice AVE de 0,480. Nenhum dos indicadores obteve um resultado inferior a 0,5 na confiabilidade dos indicadores. Pelo critério Fornell-Larcker, a raiz quadrada do AVE de cada variável latente apresentou-se maior que a correlação com todas as outras variáveis latentes. $\mathrm{Na}$ análise Cross-loadings, nenhum indicador apresentou uma correlação maior com uma variável latente do que com a sua respectiva variável latente.

Amato, Vinzi e Tenenhaus apud Tenenhaus e outros (2005) propuseram um critério de medida do ajuste global para o PLS como sendo a média geométrica 


\section{Tabela 3 - Raiz quadrada do AVE e correlações entre as variáveis latentes}

\begin{tabular}{|c|c|c|c|c|c|c|c|c|c|c|c|c|c|c|}
\hline 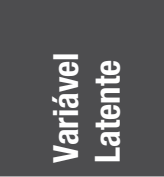 & 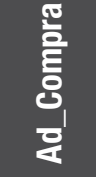 & 흘 & $\begin{array}{l}\text { 을 } \\
\text { 音 } \\
\text { 웅 }\end{array}$ & $\begin{array}{l}\text { 츨 } \\
\text { 흘 }\end{array}$ & 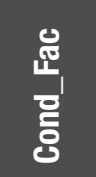 & 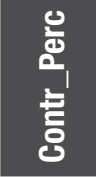 & 退 & 릉 & 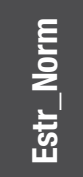 & 髉 & 흘 & 흘 & 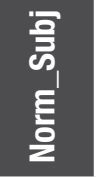 & 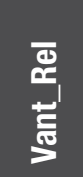 \\
\hline Ad_Compra & 0,655 & & & & & & & & & & & & & \\
\hline Atitude & 0,531 & 0,867 & & & & & & & & & & & & \\
\hline Compatib & 0,463 & 0,613 & 0,861 & & & & & & & & & & & \\
\hline Complex & 0,320 & 0,507 & 0,572 & 0,640 & & & & & & & & & & \\
\hline Cond_Fac & 0,352 & 0,464 & 0,618 & 0,579 & 0,735 & & & & & & & & & \\
\hline Contr_Perc & 0,393 & 0,460 & 0,459 & 0,570 & 0,627 & 0,815 & & & & & & & & \\
\hline Eficac & 0,386 & 0,495 & 0,588 & 0,659 & 0,709 & 0,662 & 0,790 & & & & & & & \\
\hline Envolv & 0,402 & 0,598 & 0,495 & 0,381 & 0,350 & 0,254 & 0,369 & 0,696 & & & & & & \\
\hline Estr_Norm & 0,218 & 0,187 & 0,254 & 0,037 & 0,104 & $-0,009$ & 0,057 & 0,172 & 0,818 & & & & & \\
\hline Exp & 0,237 & 0,224 & 0,231 & 0,196 & 0,206 & 0,243 & 0,226 & 0,166 & 0,084 & 0,625 & & & & \\
\hline Inov & 0,462 & 0,318 & 0,314 & 0,149 & 0,274 & 0,199 & 0,224 & 0,277 & 0,236 & 0,226 & 0,640 & & & \\
\hline Inten & 0,673 & 0,568 & 0,518 & 0,303 & 0,407 & 0,387 & 0,423 & 0,443 & 0,282 & 0,238 & 0,508 & 0,775 & & \\
\hline Norm_Subj & 0,430 & 0,344 & 0,378 & 0,169 & 0,282 & 0,232 & 0,263 & 0,285 & 0,527 & 0,141 & 0,380 & 0,505 & 0,876 & \\
\hline Vant_Rel & 0,514 & 0,644 & 0,741 & 0,601 & 0,619 & 0,434 & 0,604 & 0,545 & 0,221 & 0,204 & 0,283 & 0,522 & 0,390 & 0,806 \\
\hline
\end{tabular}

\section{Tabela 4 - Modelo geral 2}

\begin{tabular}{|l|c|c|c|}
\hline \multicolumn{1}{|c|}{ Variável latente } & AVE & Raiz quadrada do AVE & Confiabilidade composta \\
\hline Ad_Compra & 0,512 & 0,715 & 0,805 \\
\hline Atitude & 0,752 & 0,867 & 0,924 \\
\hline Compatib & 0,742 & 0,861 & 0,920 \\
\hline Complex & 0,732 & 0,855 & 0,845 \\
\hline Cond_Fac & 0,540 & 0,735 & 0,824 \\
\hline Contr_Perc & 0,665 & 0,815 & 0,888 \\
\hline Eficac & 0,625 & 0,790 & 0,869 \\
\hline Envolv & 0,538 & 0,734 & 0,913 \\
\hline Estr_Norm & 0,670 & 0,818 & 0,890 \\
\hline Exp & 0,597 & 0,772 & 0,747 \\
\hline Inov & 0,480 & 0,693 & 0,818 \\
\hline Inten & 0,600 & 0,775 & 0,853 \\
\hline Norm_Subj & 0,768 & 0,876 & 0,930 \\
\hline Vant_Rel & 0,650 & 0,806 & 0,881 \\
\hline
\end{tabular}


entre a comunalidade média e a média R2. Aplicando-se esse critério, foi verificada uma melhora no ajuste global (GoF) dos dados da Análise Geral 1 e da Análise Geral 2, passando de 0,526 para 0,550.

\section{Avaliação do modelo estrutural}

Para Tenenhaus e outros (2005), o principal critério de avaliação da confiabilidade e da validação do modelo estrutural é por meio do coeficiente de determinação R2 para as variáveis latentes endógenas. Chin (1998) considera os valores de R2 0.67, 0.33 e 0.19 no PLS como substancial, moderado e fraco, respectivamente. As variáveis latentes endógenas Contr_Perc, Atitude, Inten e Ad_Compra obtiveram valores de R2 entre moderados e substanciais, enquanto a variável Norm_Subj obteve valor de R2 entre fraco e moderado.

Em vez de considerar uma distribuição assumida dos dados, a reamostragem permite calcular uma distribuição empírica de parâmetros estimados (HAIR e outros, 1998). Por meio da reamostragem, são criadas múltiplas amostras da amostra original e calculados intervalos de confiança da distribuição de parâmetros estimados. Um dos métodos de reamostragem é o bootstrapping, utilizado na modelagem de caminhos PLS, que obtém sua amostra via amostragem com reposição da amostra original. O modelo proposto foi estimado utilizando-se a técnica bootstrapping, comparando a amostra original com as amostras geradas por essa técnica. Foram geradas 300 amostras e realizado o teste T, conforme a Tabela 5 a seguir:

O resultado mostrou que, com exceção do caminho Exp -> Atitude, os demais não apresentaram diferença entre a amostra original e as subamostras geradas pela técnica estatística com $\mathrm{p}<0,001$ ou $\mathrm{p}<0,01$.

A intenção, como antecedente da adoção da compra, teve, além de um coeficiente significativo, uma carga elevada $(0,648)$, mostrando a forte relação entre a intenção e a adoção da compra pela internet, o que confirma o modelo TPB de Ajzen e Fishbein e é similar ao modelo de Rogers, em que a decisão que forma a intenção precede a implementação.

Dos antecedentes da intenção, a atitude teve a maior carga $(0,335)$, seguida pela inovatividade $(0,275)$, pelas normas subjetivas $(0,260)$ e pelo controle percebido $(0,115)$. Ambos os antecedentes do controle percebido tiveram coeficientes significativos e cargas consideráveis, a eficácia teve maior carga $(0,438)$ que condições facilitadoras $(0,316)$.

\section{Tabela 5 - Bootstrapping modelo geral 2}

\begin{tabular}{|c|c|c|c|c|c|}
\hline Caminho & $\begin{array}{c}\text { Média da } \\
\text { amostra original }\end{array}$ & $\begin{array}{c}\text { Média das } 300 \\
\text { amostras geradas }\end{array}$ & Erro padrão & Estatistica T & $p$ \\
\hline Inten ->Ad_Compra & 0,648 & 0,649 & 0,029 & 22,449 & *** \\
\hline Atitude -> Inten & 0,335 & 0,335 & 0,047 & 7,064 & *** \\
\hline Inov -> Inten & 0,275 & 0,277 & 0,042 & 6,525 & *** \\
\hline Norm_Subj -> Inten & 0,26 & 0,26 & 0,04 & 6,503 & *** \\
\hline Contr_Perc -> Inten & 0,115 & 0,113 & 0,042 & 2,709 & $\star \star$ \\
\hline Envolv -> Atitude & 0,313 & 0,316 & 0,042 & 7,468 & *** \\
\hline Vant_Rel -> Atitude & 0,246 & 0,253 & 0,052 & 4,741 & *** \\
\hline Compatib -> Atitude & 0,194 & 0,179 & 0,057 & 3,403 & *** \\
\hline Complex -> Atitude & 0,118 & 0,119 & 0,044 & 2,644 & $\star \star$ \\
\hline Exp -> Atitude & 0,055 & 0,058 & 0,029 & 1,877 & \\
\hline Eficac -> Contr_Perc & 0,438 & 0,436 & 0,049 & 8,975 & *** \\
\hline Cond_Fac -> Contr_Perc & 0,316 & 0,32 & 0,049 & 6,46 & $* * *$ \\
\hline Estr_Norm -> Norm_Subj & 0,527 & 0,526 & 0,034 & 15,431 & *** \\
\hline
\end{tabular}

Sendo ${ }^{\star \star \star}=p<0,001,{ }^{\star \star}=p<0,01 e^{*}=p<0,05$ 
A identificação da maneira como as atitudes são formadas, fornecida pelo modelo, foram as seguintes: o envolvimento teve a maior carga $(0,313)$ entre os antecedentes da atitude, seguido pelas vantagens relativas $(0,246)$, pela compatibilidade $(0,194)$ e pela complexidade $(0,118)$. Entre esses três últimos construtos, a maior carga de vantagens relativas confirmou estudos anteriores de Taylor e Todd (1995) e Moore e Bensabat (1991).

A experiência com a internet obteve coeficiente baixo, demonstrando pouca influência sobre a atitude e não confirmando os estudos de Wolfinbarger e Gilly (2001), Huang (2003), Novak, Hoffman e Yung (2000), Johnson e outros (2004), Xia (2002) e Korzaan (2003).

O modelo obteve um índice de explicação de variância da adoção da compra de $42,0 \%$ e de intenção de $50,5 \%$, que supera o nível de $40 \%$, que, segundo Venkatesh e outros (2003), normalmente é encontrado na intenção nos modelos que estudam a aceitação de tecnologia.
A Figura 4 a seguir apresenta os resultados aplicados ao modelo integrativo teórico proposto.

\section{CONCLUSÕES}

Desde o início dos anos 1990, tem ocorrido um crescimento acelerado do comércio eletrônico. Novas empresas nasceram, trazidas pelas oportunidades que se abriram com a nova tecnologia. Por outro lado, empresas líderes de mercado estão sendo ameaçadas se não se posicionarem em relação ao novo canal que está se consolidando, e empresas não líderes estão, por meio da nova tecnologia, encontrando caminhos potenciais para seu crescimento.

Do lado do consumidor, esse novo canal está se apresentando de modo diferente em relação aos canais tradicionais, trazendo-lhe novas formas de compra, vantagens e riscos. No processo de compra, o consumidor

\section{Figura 4 - Modelo integrativo}

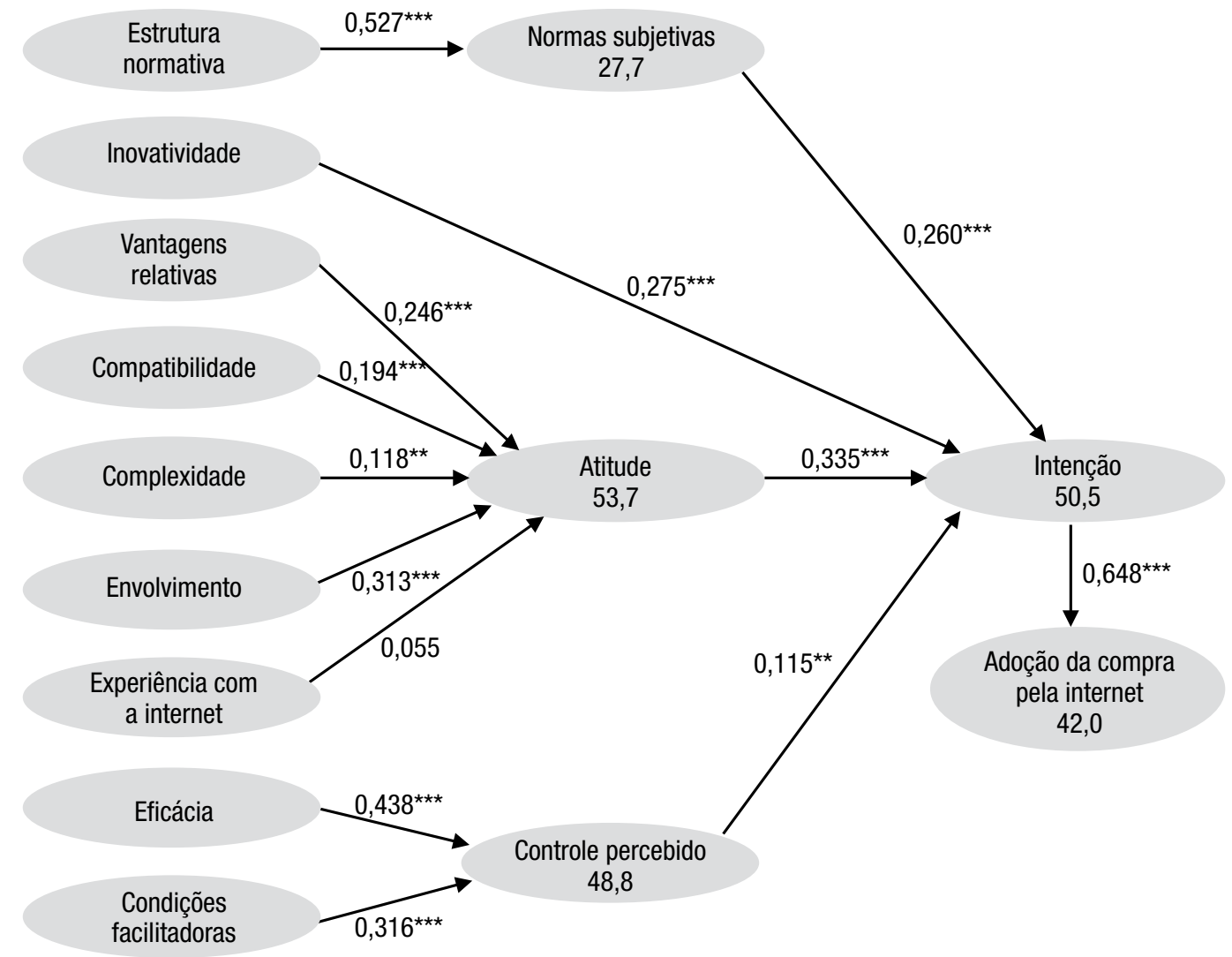


é estimulado por fatores culturais, sociais, pessoais e psicológicos, por estímulos de marketing e por outros estímulos, e todos estão mudando com a nova tecnologia; as diferenças entre as lojas virtuais e as lojas físicas, além das estratégias inovadoras de marketing, estão mudando os hábitos e estilos de comprar. O processo de decisão de compra do consumidor está se adaptando às facilidades trazidas pela internet, que tem recebido uma adoção crescente e contínua.

O propósito deste trabalho foi estudar a influência da inovatividade, do envolvimento, da atitude e da experiência com a internet na adoção da compra pela internet, por meio de um modelo teórico integrativo.

Uma pesquisa efetuada com 474 respondentes que compraram pela internet foi utilizada para testar as relações entre os fatores, por meio de equações estruturais. O modelo proposto, contendo os fatores apontados, obteve resultados superiores aos que geralmente são obtidos nos estudos que analisam a adoção de uma inovação, o que mostra que o conjunto de variáveis escolhidas, com exceção da experiência com a internet, contribui para um melhor entendimento dos fatores que levam à adoção da compra pela internet.

- Os resultados apontaram que:

- A adoção da compra pela internet é diretamente influenciada pela intenção.

- Os antecedentes que formam a intenção foram, em ordem de importância, a atitude, a inovatividade, as normas subjetivas e o controle percebido.

- A atitude, por sua vez, é influenciada pelo envolvimento, pelas vantagens relativas, pela compatibilidade e pela complexidade.

- O controle percebido é influenciado pela eficácia e pelas condições facilitadoras.

- Não foi encontrada influência entre a experiência com a internet e a compra pela internet.

Estes resultados implicam que a adoção da compra pela internet é significantemente relacionada com a inovatividade, o envolvimento e a atitude.

Com base nas relações entre as variáveis e nos resultados obtidos, é possível desenvolver programas para encorajar os consumidores a adotar a compra on-line.

Por exemplo: o resultado sugere que a formação de crenças e de sentimentos sobre a compra pela internet (atitude) tem um efeito forte sobre sua intenção. Os resultados da relação entre normas subjetivas e atitude mostram a influência da pressão social sobre o indivíduo com relação à compra pela internet, indicando que as pessoas procuram corresponder às expectativas do seu meio social, o que é contrário às considerações de Bobbitt e Dabholkar (2001) em seu modelo integrativo. Isso fornece elementos para estratégias promocionais de marketing enfatizando a adoção da compra pela internet. A relação positiva entre o controle percebido e a intenção indica que o indivíduo acredita que possui condições de realizar a compra pela internet se desejar e que a percepção de que a eficácia e os recursos necessários são importantes para a compra on-line.

O envolvimento tem como construto básico o estado motivacional, os resultados mostram a força entre a atenção despertada pela compra on-line e a atitude em relação a ela, podendo encorajá-la e podendo diminuir a resistência em adotá-la.

As vantagens relativas, como segundo fator, podem ser utilizadas para incentivar a adoção da compra pela internet, acentuando suas vantagens. A compatibilidade com o estilo de vida refere-se à crença que o indivíduo tem de que a compra pela internet é compatível com seu estilo de vida; isso pode ser utilizado em comunicações dirigidas incentivando seu uso ou disponibilizando formas facilitadoras e acessíveis de adotá-la.

A inovatividade como preditora da intenção confirmou estudos anteriores de Alcañiz e outros (2008), Manzano e outros (2009) e Citrin e outros (2000), apresentando informações que possibilitam estratégias de marketing. O mix de marketing pode ser estabelecido de modo a considerar o perfil desses primeiros adotantes. O controle da satisfação desses primeiros usuários pode servir para a difusão da adoção da compra on-line pelos seguidores por meio do boca a boca.

A experiência com a internet obteve coeficiente baixo, demonstrando pouca influência sobre a atitude e não confirmando os estudos anteriores, o que mereceria uma investigação específica.

Espera-se que este trabalho ajude a conhecer um pouco melhor o consumidor em relação à compra pela internet e estimule o interesse pela pesquisa sobre o tema, incentivando o trabalho de novos textos acadêmicos.

Os resultados deste trabalho são limitados pela amostra, pelos fatores e pelas medidas utilizadas que se procurou relacionar com a compra on-line.

A amostra não foi probabilística, o que não possibilita a generalização dos resultados da pesquisa, limitando-a à amostra estudada. A maioria dos componentes da amostra considerada contemplou universitários, o que não representa a maior parte da população. Estudos de outras populações, com pouca variação das dimen- 
sões culturais e variando somente uma delas, poderiam ajudar a isolar o efeito de cada uma dessas variáveis e enriqueceriam os resultados obtidos.

As escalas e medidas utilizadas podem não contemplar o alcance dos fatores que se queria medir. Poderiam ser feitas pesquisas utilizando outras medidas e escalas para confirmar e expandir os resultados deste estudo.

Os fatores considerados não tinham como objetivo nem poderiam esgotar o assunto, diversos outros fatores colocados na revisão bibliográfica poderiam ser introduzidos neste modelo e também ampliar seus resultados.

Outra limitação diz respeito ao autopreenchimento das variáveis, que podem introduzir erros nos dados. As limitações de recursos e de tempo impossibilitaram a utilização de métodos mais precisos no levantamento dos dados.

A autoavaliação é também uma limitação que, em alguns casos, pode confundir valores reais com valores que se gostaria de ter.

São necessários mais esforços de pesquisas para melhor entender a natureza do processo e da experiência da compra on-line.

Futuros estudos poderiam ampliar os resultados obtidos no modelo proposto neste trabalho, incluindo outros fatores como cultura, subculturas, fatores sociais, fatores pessoais, outros fatores psicológicos e situacionais.

A análise poderia também ser realizada por categoria de produtos ou serviços na compra on-line em que os fatores e sua influência poderiam fornecer resultados diferentes.

O estudo da experiência com a internet e da compra pela internet é outro assunto de interesse que merece ser mais aprofundado, considerando outra escala para sua caracterização.

\section{REFERÊNCIAS}

AJZEN, I. From intentions to actions: a theory of planned behavior. In: J. Kuhl and J. Beckmann (Eds), Action control from cognition to behavior. New York: Springer Verlag, p. 11-39, 1985.

ALCAÑIZ, E. B; MAFÉ, C. R; MANZANO, J. A. J; BLAS, S. $\mathrm{S}$. Influence of on-line shopping information dependency and innovativeness on internet shopping option. On-line Information Review, v. 32, n. 5, p. 648-667, 2008.
AUN, F. Brazil, Russia, India and China to lead internet growthb through 2001. The ClickZ Network, 2007. Disponível em: http://www.ecominfocenter.com/index. html? page $=/$ infosources $/$ websites $/$ statistics.html. Acesso em 07.10.2007.

BEARDEN, W. O; NETEMEYER, R. G. Handbook of marketing scales. 2nd ed. California: Sage Publications, 1999.

BIENSTOCK, C. C; STAFFORD, M. R. Measuring involvement with the service: a further investigation of scale validity and dimensionality. Journal of Marketing Theory and Practice, v. 14, n. 3, p. 209-221, 2006.

BLACKWELL, R. D; MINIARD, P. W; ENGEL, J. F. Comportamento do consumidor. São Paulo: Pioneira Thomson Learning, 2005.

BLAKE, B. F; NEUENDORF, K. A; VALDISERRI, C. M. Innovativeness and variety of internet shopping. Internet Research, v. 13, n. 3, p. 156-164, 2003.

BOBBITT, L. M; DABHOLKAR, P. A. Integrating attitudinal theories to understand and predict use of tecgnology-based self-service: the internet as an illustration. International Journal of Service Industry Management, v. 12, n. 5, p. 423-450, 2001.

CHIN, W. W. The partial least squares approach to structural equation modeling. In: MARCOULIDES, G. A. (Ed). Modern methods for business research. Mahwah: Lawrence Erlbaum Associates, 1998. p. 295-358.

$\mathrm{CHO}, \mathrm{J}$. Likelihood to abort an on-line transaction: influences from cognitive evaluations, attitudes, and behavioral variable. Information \& Management, v. 41, n. 7, p. 827838, 2004.

CITTRIN, A. V; SPROTT, D. E; SILVERMAN, S. N; STEM JR, D. E. Adoption of internet shopping: the role of consumer innovativeness. Industrial Management \& Data Systems, v. 100, n. 7, p. 294-300, 2000.

DAVIS, F. D. Perceived usefulness, perceived ease of use, and user acceptance of information technology. MIS Quarterly, v. 13, n. 3, p. 319-340. 1989.

EASTLICK, M. A.; LOTZ, S. Profiling potential adopters and non-adopters of an interactive electronic shopping medium. International Journal of Retail \& Distribution Management. Vol. 27, n. 6; p. 209-228, 1999. 
ENGEL, J. F; BLACKWELL, R. D; MINIARD, P. W. Comportamento do consumidor. 8. ed. Rio de Janeiro: LTC, 2000.

GEFEN, D; KARAHANNA, E; STRAUB, D. W. Trust and TAM in on line shopping: an integrated mode. MIS Quarterly, v. 27 , n. 1, p. 51-90, 2003.

GOLDSMITH, R. E. Using the domain specific innovativeness scales to identify innovative internet consumers. Internet Research, v. 11, n. 2, p. 149-158, 2001.

GOLDSMITH, R. E; FLYNN, L. R. Psychological and behavioural drivers of on-line clothing purchase. Journal of Fashion Marketing and Management, v. 8, n. 1, p. 84-95, 2004.

GOLDSMITH, R. E; HOFACKER, C. F. Measuring consumer innovativeness. Journal of the Academy of Marketing Science, v. 19, n. 3, p. 209-221, 1991.

HAIR JR, J. F; ANDERSON, R. E; TATHAM, R. L; BLACK, W. C. Multivariate data analysis. 5th ed. New Jersey: Prentice Hall, 1998.

HAIR JR, J. F; ANDERSON, R. E; TATHAM, R. L; BLACK, W. C. Multivariate data analysis. 7th ed. New Jersey: Prentice Hall, 2009.

HAWKINS, D. L; MOTHERSBAUGH, D. L; BEST, R. J. Comportamento do consumidor. construindo a estratégia de marketing. Tradução da 10. ed. Rio de Janeiro: Elsevier, 2007.

HENSELER, J; RINGLEAND, C. M.; SINKOVICS, R. R. The use of partial least squares path modeling in international marketing. New Challenges to International Marketing Advances in International Marketing, v. 20, Part IV, p. 277-319, 2009.

HUANG, M. H. Modeling virtual exploratory and shopping dynamics: an environmental psychology approach. Information E Management, v. 41, n. 1, p. 39-47, 2003.

IM, S; BAYUS, B. L; MASON, C. H. An empirical study of innate consumer innovativeness, personal characteristics, and new-product adoption behavior. Journal of the Academy of Marketing Science. v. 31, n. 1, p. 61-73, 2003.

INTERNET WORLDSTATS. Internet usage statistics: the big picture. 2011. Disponível em: http://www.internetworldstats.com/stats.htm. Acesso em 06.07.2011.
JOHNSON, E. J; MOE, W. W; FADER, P. S; BELLMAN, S; LOHSE, G. L. On the depth and dynamics of on-line search behavior. Management Science, v. 50, n. 3, p. 299-309, 2004.

JOSEPH, B; VYAS, S. J. Concurrent validity of a measure of innovative cognitive style. Academy of Marketing Science, v. 12 , n. $1 / 2$, p. $159-175,1984$.

KARAHANNA, E; STRAUB, D. W; CHERVANY, N. L. Information technology adoption across time: a cross-sectional comparison of pre-adoption and post-adoption beliefs. MIS Quarterly, v. 23, n. 2, p. 183-213, 1999.

KORZAAN, M. L. Going with the flow: predicting on-line purchase intentions. Journal of Computer Information Systems, v. 43, n. 4, p. 25-31, 2003.

LIMEIRA, T. M. V. E-marketing: o marketing na internet com casos brasileiros. 2. ed. São Paulo: Saraiva, 2007.

MAHAJAN, V; MULLER, E; BASS, F. M. New product diffusion models in marketing: a review and directions for research. Journal of Marketing, v. 54, n. 1, p. 1-25, 1990.

MANZANO, J. A; NAVARRÉ, C. L; MAFÉ, C. R; BLAS, S. S. The role of consumer innovativeness and perceived risk in on-line banking usage. International Journal of Bank Marketing, v. 27, n. 1, p. 53-75, 2009.

MATHIESON, K; PEACOCK, E; CHIN, W. Extending the technology acceptance model: the influence of perceived user resources. The Database for Advances in Information Systems, v. 32, n. 3, p. 86-112, 2001.

MCQUARRIE, E. F; MUNSON, J. M. The Zaichkowsky personal involvement inventory: modification and extension. Advances in Consumer Research, v. 14, n. 1, p. 36-40, 1987.

MOORE, G; BENSABAT, I. Development of an instrument to measure the perceptions of adopting an information technology innovation. Information Systems Research, v. 2, n. 3, p. 192-222, 1991.

NOVAK, T. P; HOFFMAN, D. L; YUNG, Y. Measuring the customer experience in on line environments: a structural modeling approach. Marketing Science, v. 19, n. 1, p. 22-42, 2000 . 
PHAU, I; LO, C. Profiling fashion innovators: a study of self-concept, impulse bying and Internet purchase intent. Journal of Fashion Marketing and Management, v. 8, n. 4, p. 399-411, 2004.

ROGERS, E. M. Diffusion of innovations. 5 th ed. New York: Free Press, 2003.

SHIH, Y; FANG, K. The use of a decomposed theory of planned behavior to study Internet banking in Taiwan. Internet Research, v. 14, n. 3, p. 213-223, 2004.

SMITH, A. D; RUPP, W. T. Strategic on line customer decision making: leveraging the transformational power of the Internet. On Line Information Review, v. 27, n. 6, p. 418-432, 2003.

TAYLOR, S; TODD, P. Decomposition and crossover effects in the theory of planned behavior: a study of consumer adoption intentions. International Journal of Research in Marketing, v. 12, n. 2, p. 137-155, 1995.

TENENHAUS, M; VINZI, V. E; CHATELIN, Y. M; LAURO, C. A global goodness-of-fit index for PLS structural equation modeling. Oral comunication to PLS Club, HEC School of Management, France, 2005.

TURBAN, E; MCLEAN, E; WETHERBE, J. Tecnologia da informação para gestão: transformando os negócios na economia digital. 3. ed. Porto Alegre: Bookman, 2004.

U. S. CENSUS BUREAU NEWS. U. S. Quaterly Retail E-Commerce Sales. Department of Commerce. Washington, D. C, D. C, May 16, 2011. Disponível em: http://www.census. gov/retail/. Acesso em 06.07.2011.

VENKATESH, V; MORRIS, M. G; DAVIS, G. B; DAVIS, F. D. User acceptance of information technology: toward a unified view. MIS Quarterly, v. 27, n. 3, p. 425-478, 2003.

WOLFINBARGER, M; GILLY, M. Shopping on-line for freedom, control, and fun. California Management Review, v. 43, n. 2, p. 34-55, 2001.

WU, S. Internet marketing involvement and consumer behavior. Asia Pacific Journal of Marketing and Logistics, v. 14, n. 4, p. 36-53, 2002.

XIA, L. Affect as information: the role of affect in consumer on-line behaviors. Advances in Consumer Research, v. 29, n. 1, p. 93-100, 2002.
ZAICHKOWSKY, J. L. The personal involvement inventory: reduction, revision, and application to advertising. Journal of Advertising, v. XXIII, n. 4, p. 59-70, 1994.

ZAICHKOWSKY, J. L. Conceptualizing involvement. Journal of Advertising, v. 15, n. 2, p. 4-34, 1986. 